\title{
Proinflammatory and anti-inflammatory cytokine responses in preterm infants with systemic infections
}

\author{
P C Ng, K Li, R P O Wong, K Chui, E Wong, G Li, T F Fok
}

Arch Dis Child Fetal Neonatal Ed 2003;88:F209-F213

See end of article for authors' affiliations

Correspondence to: Professor $\mathrm{Ng}$, Department of Paediatrics, Level 6, Clinical Sciences Building, Prince of Wales Hospital, Shatin, NT, Hong Kong; pakcheungng@cuhk.edu.hk

Accepted 5 August 2002

\begin{abstract}
Objective: A prospective study to investigate the pattern of proinflammatory and anti-inflammatory cytokine responses in preterm infants with systemic infection.

Methods: Very low birthweight infants in whom infection was suspected when they were $>72$ hours of age were eligible. A full sepsis screen was performed in each episode. Key cytokines of both proinflammatory and anti-inflammatory pathways, including interleukin (IL) 2, IL4, IL5, IL6, IL 10, interferon (IFN) $\gamma$, and tumour necrosis factor (TNF) $\alpha$, were measured at 0 (at the time of sepsis evaluation), 24, and 48 hours by flow cytometric analysis or immunoassay.

Results: Thirty seven of the 127 episodes of suspected clinical sepsis were proven infection or necrotising enterocolitis. Both proinflammatory (IL2, IL6, IFN $\gamma$, TNF $\alpha$ ) and anti-inflammatory (IL4, IL 10) cytokines were significantly increased in infected infants compared with non-infected infants. Significant correlations were observed between IL6 and TNF $\alpha$ or IL 10 as well as IL 10 and IFN $\gamma$ in infected infants. In the subgroup analysis, plasma IL6, IL 10, and TNF $\alpha$ concentrations, and IL10/TNF $\alpha$ and IL6/ILI0 ratios were significantly elevated in patients with disseminated intravascular coagulation compared with infected infants without. The IL10/TNF $\alpha$ ratios had decreased significantly 48 hours after the onset, whereas the IL6/IL 10 ratio showed only a non-significant decreasing trend. Further, the IL6/IL 10 ratio in the deceased infant was disproportionally increased at presentation and continued to increase despite treatment.

Conclusion: The results indicate that the counter-regulatory mechanism between the proinflammatory and anti-inflammatory cytokine pathways is probably operational in preterm infants of early gestation. High plasma IL6, IL 10, and TNF $\alpha$ concentrations, and IL 10/TNF $\alpha$ and IL6/IL10 ratios signify severe infection, but transiently elevated plasma IL 10 concentration or IL 10/TNF $\alpha$ ratio does not necessarily indicate a poor prognosis.
\end{abstract}

C ytokines are endogenous chemical mediators which play an important role in orchestrating the inflammaammatory and anti-inflammatory cytokines is strictly controlled by complex feedback mechanisms. ${ }^{2-6}$ Proinflammatory cytokines are primarily responsible for initiating an effective defence against exogenous pathogens. However, overproduction of these mediators can be harmful and may ultimately lead to shock, multiple organ failure, and death. ${ }^{78}$ In contrast, anti-inflammatory cytokines are crucial for downregulating the exacerbated inflammatory process and maintaining homoeostasis for proper functioning of vital organs, ${ }^{9}{ }^{10}$ but excessive anti-inflammatory response may also result in the suppression of body immune function. ${ }^{11}{ }^{12}$ The pattern of circulating cytokines and the pathogen characteristics and load may determine the differentiation of precursor $\mathrm{T}$ helper (Th0) lymphocytes into Thl and Th2 cells. ${ }^{13}$ Thl cells, which produce interferon (IFN) $\gamma$, interleukin (IL) 2, and tumour necrosis factor (TNF) $\beta$, are known to promote cell mediated immunity and phagocytic activity, especially during intracellular infections, ${ }^{12}$ whereas Th2 cells, which produce IL4, IL5, IL6, IL9, IL10 and IL13, support humoral immunity and antibody production, ${ }^{12}$ and play a dominant role in allergic $^{14}$ and stress responses. ${ }^{15}$ Although the cytokine patterns of lymphocyte subsets have been extensively studied in adult patients with infection and septic shock, ${ }^{346}$ the exact mechanism of regulation and their effects on clinical outcomes remain to be elucidated. Furthermore, little is known about the profile of proinflammatory and antiinflammatory cytokines in septic preterm, very low birthweight (VLBW) infants, in whom the immune system has been considered to be suboptimal and immature. ${ }^{16}$ Recent advances in flow cytometry have permitted quantitative measurement of key cytokines, including IL2, IL4, IL5, IL10, IFN $\gamma$, and TNF $\alpha$, with a minimal volume of plasma $(0.05 \mathrm{ml})$, and this new technology would be most suited for assessing the inflammatory cascade in preterm infants. This prospective study was thus designed to investigate the relation between proinflammatory and anti-inflammatory cytokines in VLBW infants with bacterial septicaemia, systemic fungal infection, and necrotising enterocolitis (NEC) using flow cytometry. The impact of the severity of disease and the type of infection on the cytokine cascade was also studied.

\section{PATIENTS AND METHODS \\ Patients}

Preterm infants with (a) birth weight $<1500 \mathrm{~g}$, $(b)$ postnatal age $>72$ hours, (c) sign and symptoms suggestive of systemic infection and requiring full sepsis evaluation and antibiotic treatment, and $(c)$ parental consent, in the neonatal unit at Prince of Wales Hospital, Hong Kong were eligible for enrollment into the study. Patients who were already on parenteral antibiotic treatment at the time of sepsis evaluation, or had severe congenital or chromosomal abnormalities, were excluded. Recruitment of suspected infection episode was carried out prospectively over a 22 month period.

Abbreviations: IFN, interferon; IL, interleukin; TNF, tumour necrosis factor; VLBW, very low birthweight; NEC, necrotising enterocolitis; CRP, $C$ reactive protein; DIC, disseminated intravascular coagulation 


\section{Methods}

Signs and symptoms suggestive of clinical sepsis have been described in detail in our previous study. ${ }^{17}$ All infants were recruited at the time of evaluation for suspected clinical sepsis. In each episode, a full sepsis screen was performed, which included: cerebrospinal fluid, blood, urine, stool, and endotracheal aspirate (infants on ventilator) cultures for bacteria and fungi; removal and culture of indwelling central lines or catheters; and cultures of specific sites and surgical specimens such as peritoneal fluid, abscess, and biopsy specimen. Chest radiograph was routinely performed during the initial screening procedure, and an abdominal radiograph was requested when patients presented with signs suggestive of intraabdominal pathology. Haematological and biochemical laboratory investigations, including a complete blood count, differential white cell and platelet counts, arterial blood gas, and serum glucose concentration, were also performed. In addition to our routine serial $\mathrm{C}$ reactive protein (CRP) measurements, blood specimens were also obtained for evaluation of IL2, IL4, IL5, IL6, IL10, IFN $\gamma$, and TNF $\alpha$. The first sample was taken at the time of the initial sepsis evaluation $(0$ hour), and two further samples were obtained at 24 and 48 hours after the onset for monitoring the clinical progress. This schedule of blood sampling coincided with our unit policy for serial blood count and CRP measurements after a suspected episode of infection had been identified. Intravenous broad spectrum antibiotics were started immediately after the sepsis screening. Three categories of "infective" episodes were prospectively defined. ${ }^{17}$ Briefly, they were as follows.

Group 1, the "infected group" which consisted of episodes that had been confirmed as septicaemia, meningitis, pneumonia, peritonitis, systemic fungal infection, or NEC (stage II or above in Bell's classification). A subgroup of severely infected infants who developed disseminated intravascular coagulation (DIC) and clinically presented with elevated serum D-dimer concentration $>1.0 \mu \mathrm{g} / \mathrm{ml}$ (normal range $0.5-1.0$ $\mu \mathrm{g} / \mathrm{ml}$ ), thrombocytopenia $<50 \times 10^{9} / \mathrm{l}$ (normal range $>150 \times$ $\left.10^{9} / 1\right)$, and deranged coagulation with prolonged activated partial thromboplastin time $>120$ seconds (normal range 26.2-40.1 seconds) was also identified.

Group 2 was defined as the "non-infected group" and consisted of episodes that met the screening criteria for suspected clinical sepsis but were subsequently proven not to be infected. ${ }^{17}$

Group 3 was the "comparison group" and consisted of blood samples taken once from 20 well, VLBW infants between week 1 and 5 of postnatal age for CRP and cytokine measurements. The collection of the latter blood samples coincided with the weekly monitoring of haemoglobin, liver function, and bone profile of preterm infants.

\section{Measurement of cytokines and CRP}

Blood samples collected from indwelling arterial lines or venepunctures were immersed in ice and immediately transported to the laboratory for processing. Plasma was separated by centrifugation ( $1900 \mathrm{~g}$ for five minutes) at $4^{\circ} \mathrm{C}$ and stored in $200 \mu \mathrm{l}$ aliquots at $-80^{\circ} \mathrm{C}$ until analysis. CRP was measured by a turbidity assay against control standards, as specified by the manufacturer (Behring Diagnostics Inc, Westwood, Massachusetts, USA). IL6 was measured by the enzyme linked immunoassay technique using a commercially available kit (R\&D System Inc, Minneapolis, Minnesota, USA). The sensitivity of the IL6 assay, as defined by the corresponding concentration at two standard deviations above the mean measurement of the negative control $(0 \mathrm{pg} / \mathrm{ml})$ is $0.70 \mathrm{pg} / \mathrm{ml}$.

A panel of Th1 and Th2 cytokines including IL2, IL4, IL5, IL10, INF $\gamma$, and TNF $\alpha$ were simultaneously quantified by the Human Thl/Th2 Cytokine Cytometric Bead Array Kit (BD Pharmingen, San Diego, California, USA) using flow cyto- metry. This assay kit provided a mixture of six micro-bead populations with distinct fluorescent intensities (FL-3) and were precoated with capture antibodies specific for the Th1/Th2 proteins. When the beads were incubated with the corresponding phycoerythrin conjugated detection antibodies and the test sample, sandwich complexes were formed. The assay sensitivities for IL2, IL4, IL5, IL10, TNF $\alpha$, and IFN $\gamma$ were $2.6,2.6,2.4,2.8,2.8$, and $7.1 \mathrm{pg} / \mathrm{ml}$ respectively. For our analysis, $50 \mu \mathrm{l}$ plasma or the provided standard cytokines were added to the pre-mix micro-beads in $12 \mathrm{~mm} \times 75 \mathrm{~mm}$ Falcon tubes. After the addition of $50 \mu \mathrm{l}$ detecting reagent, the mixture was incubated for three hours in the dark at room temperature. This mixture was washed and centrifuged at 200 $g$ for five minutes and the pellet was resuspended in $300 \mu \mathrm{l}$ washing buffer. The FACS Calibur flow cytometer (BD Pharmingen) was calibrated with the Setup Beads, and 3000 events were acquired for each sample. The quantities of individual cytokines as indicated by their fluorescent intensities (FL-2) were computed using the standard reference curve of the CellQuest and CBA Software (BD Pharmingen).

\section{Statistical analysis}

The basic data and the plasma concentration of different cytokines in the infected (group 1: 0 hour), non-infected (group 2: 0 hour), and comparison (group 3) group were compared using the Kruskal-Wallis test and $\chi^{2}$ test. The Mann-Whitney $\mathrm{U}$ test was also used to compare the corresponding plasma cytokine concentrations between the infected and noninfected infants at 24 and 48 hours, and in the subgroup analysis of infected patients. In addition, the relation between different cytokines was assessed by the Spearman's correlation. The statistical analysis was performed on raw or logarithmically transformed data where appropriate. All statistical tests were performed by SPSS for Windows (Release 10; SPSS Inc, Chicago, Illinois, USA). The level of significance was set at $5 \%$ in all comparisons.

\section{Ethical approval}

The study was approved by the research ethics committee of the Chinese University of Hong Kong. Informed consent was obtained from the parents or guardians for all patients.

\section{RESULTS}

There were no significant differences in gestational age (median (interquartile range) $29.2(27.8-31.2) v 28.6$ (27.1$30.1)$ v 29.1 (27.5-31.2) weeks), birth weight (median (interquartile range) $1183(864-1048) v 1131$ (903-1292) $v 1180$ (845-1361) g), Apgar scores at one and five minutes, and male to female ratio between the infected, non-infected, and comparison groups respectively. A total of 127 episodes of suspected clinical sepsis were investigated in 80 VLBW infants. One, one, seven, and 26 patients had a sepsis screen performed five, four, three, and two times respectively. The remaining 45 infants received only one sepsis screen. Hence, the results are based on the number of suspected infection episodes rather than the number of infants recruited into the study. Thirty seven of the 127 episodes of suspected clinical sepsis were confirmed infection or NEC. Table 1 gives details of the clinical pathologies of infected infants and causative organisms. No micro-organisms could be isolated from the blood and cerebrospinal fluid in four infected cases. These patients presented with unstable temperature, hypotension, recurrent apnoea, and severe desaturation. All were judged to be genuinely septic based on their strong and persistent signs of infection, and they were subsequently found to have raised CRP and at least two other markers of infection. These infants received a full course of antibiotic treatment, and their clinical improvement bore close temporal relation to the normalisation of infection markers. Thus we believe that these cases have not been misclassified. 
Table 1 Details of clinical pathologies and organisms of the infected episodes

\begin{tabular}{ll}
\hline Pathology/organism & No of episodes $(n=37)$ \\
\hline Gram positive septicaemia $(n=15(40.5 \%))$ \\
Coagulase negative staphylococci 13 (1 case of pneumonia) \\
Staphylococcus aureus & 1 \\
Enterococcus & 1 \\
Gram negative septicaemia $(n=6(16 \%))$ \\
Serratia marcescens & 2 \\
Klebsiella sp. & 3 (1 case of pneumonia) \\
Escherichia coli & 1 \\
Fungal septicaemia $(n=4(11 \%))$ & 3 (1 case of meningitis) \\
Candida albicans & 1 \\
Malassazia furfur & 8 (2 cases of bowel perforation) \\
Necrotising enterocolitis $(n=8(21.5 \%))$ & 4 \\
Unidentified organisms $(n=4(11 \%))$ & 4
\end{tabular}

\section{Overall analysis}

Plasma concentrations of IL2, IL4, IL6, IL10, IFN $\gamma$, and TNF $\alpha$ at the time of sepsis evaluation ( 0 hour) were significantly higher in the infected group than in the non-infected ( 0 hour; $\mathrm{p}<0.002)$ and comparison $(\mathrm{p}<0.05)$ groups. Similarly, these cytokines (except TNF $\alpha$ at 48 hours) were also significantly raised at 24 and 48 hours in infected compared with non-infected infants $(\mathrm{p}<0.04$, table 2$)$. As expected, no significant difference in plasma cytokine concentrations was observed between non-infected infants at 0 hour and infants in the comparison group. In addition, plasma IL5 concentrations did not differ significantly at any time points between the three groups.

There was significant correlation between different cytokines in infected infants. In particular, IL6 consistently correlated significantly with TNF $\alpha(r>0.36, \mathrm{p}<0.03)$ and IL10 $(r>0.49, \mathrm{p}<0.002)$ throughout the study period, whereas IL10 also significantly correlated with IFN $\gamma(r>0.43$, $\mathrm{p}<0.015$ ) at 0 and 24 hours.

\section{Subgroup analysis}

The infected infants (group 1) were further divided into clinical subgroups for analysis. They were $(a)$ infants with NEC $v$ those without, $(b)$ infants with DIC $v$ those without, and $(c)$ infants with Gram positive organism infection $v$ those with Gram negative organism or systemic fungal infection. Table 3 summarises the cytokine results of these clinical subgroups. There was no significant difference in the peak plasma cytokine concentrations between infants who had NEC and infected infants without the disease. In contrast, the peak plasma IL6 concentrations were significantly higher in patients with Gram negative or fungal infections than in infants with Gram positive sepsis $(p=0.035)$. The peak plasma IL6, IL10, and TNF $\alpha$ concentrations were significantly elevated in septic infants with DIC compared with those without $(p<0.0001)$. Similarly, the IL10/TNF $\alpha$ and IL6/IL10 ratios - that is, the ratios used to evaluate the balance between proinflammatory and anti-inflammatory cytokines-were significantly higher in patients with DIC $(\mathrm{p}<0.01$ at 0 and 24 hours for IL10/TNF $\alpha$, and $\mathrm{p}<0.05$ at 0,24 , and 48 hours for IL6/IL10, with or without the death case). The IL10/TNF $\alpha$ ratios, however, had fallen significantly at the subsequent time points ( 24 and 48 hours) compared with the corresponding levels at 0 hour $(\mathrm{p}<0.005$ at 48 hours for infants with DIC, and $p<0.001$ at 24 and 48 hours for infected infants without DIC; fig 1), whereas the IL6/IL10 ratios did not decrease significantly during the study period (fig 2).

\section{The deceased case}

Of 37 infected infants, one patient died from fungal septicaemia and DIC. This patient had very elevated plasma IL6 ( 1875 $\mathrm{pg} / \mathrm{ml})$ and ILl0 (122.7 pg/ml) concentrations at presentation, and these levels remained high throughout the study period (plasma IL10 (80.8 and $48.8 \mathrm{pg} / \mathrm{ml}$ ) and IL6 (1855 and 5608 $\mathrm{pg} / \mathrm{ml})$ at 24 and $48 \mathrm{~h}$, respectively). In contrast with the patients with DIC who survived, the IL6/IL10 ratios of this infant continued to increase despite treatment. The log IL6/IL10 ratios were 2.7, 3.1, and 4.7, and the log IL10/TNF $\alpha$ ratios were $3.5,3.2$, and 2.8 at 0,24 , and 48 hours respectively.

\section{DISCUSSION}

Our data show activation of specific proinflammatory (IL2, IL6, IFN $\gamma$, TNF $\alpha$ ) and anti-inflammatory (IL4, IL10) cytokines in response to sepsis and successful treatment in preterm, VLBW infants. Whereas the panel of proinflammatory and anti-inflammatory cytokines were regulated in response to the nature and severity of the diseases, IL5, a cytokine principally related to allergy and hypersensitivity stress, remained largely unaffected. The reactions to sepsis were particularly prominent in the activation of IL6 and IL10, which were elevated 35 -fold and 22-fold respectively at 0 hour compared with the levels in those infants who were subsequently shown to be non-infected (table 3). Further, the cytokine levels were decreased by $83 \%$ and $87 \% 48$ hours after treatment. To date, the interaction between proinflammatory and antiinflammatory cytokines in response to sepsis remains a controversial subject. Most evidence, including our present findings, points to the operation of a feedback or counterregulatory mechanism. IL6 and TNF $\alpha$ are potent proinflammatory cytokines and are responsible for eliciting a strong inflammatory reaction, which, if left uncontrolled, may lead to severe hypotension, multiple organ dysfunction, and death. ${ }^{18}{ }^{19}$ This proinflammatory state of acute phase response to sepsis will ultimately trigger a compensatory antiinflammatory reaction involving antagonist mediators such as

Table 2 Plasma cytokine concentrations ( $\mathrm{pg} / \mathrm{ml}$ ) in the infected (group 1, $\mathrm{n}=37$ ), non-infected (group 2, $\mathrm{n=90}$ ) and comparison (group $3, n=20$ ) groups at 0,24 , and 48 hours of sepsis evaluation

\begin{tabular}{llllllll}
\hline & IL2 & IL4 & IL5 & IL6 & IL10 & IFN $\gamma$ & TNF $\alpha$ \\
\hline $\begin{array}{l}\text { O hour } \\
\text { Infected group }\end{array}$ & $2.8(0.0-5.2)$ & $3.0(0.0-5.7)$ & $4.7(2.1-17.1)$ & $140(34-1251)$ & $109(20-245)$ & $67(27-787)$ & $3.0(1.4-5.7)$ \\
$\quad$ Non-infected group & $0.0(0.0-0.8)$ & $0.0(0.0-2.2)$ & $4.1(2.6-7.6)$ & $4(2-11)$ & $5(4-9)$ & $8(0-17)$ & $2.0(0.0-2.8)$ \\
$\quad$ Comparison group & $0.0(0.0-1.6)$ & $1.6(0.0-2.4)$ & $3.1(1.8-5.7)$ & $2(1-6)$ & $7(5-8)$ & $9(0-14)$ & $2.1(0.6-2.9)$ \\
$\begin{array}{c}24 \text { hours } \\
\text { Infected group }\end{array}$ & $2.0(0.0-3.6)$ & $3.0(0.0-6.6)$ & $3.7(2.2-6.1)$ & $25(9-95)$ & $29(9-68)$ & $42(18-83)$ & $2.5(0.0-3.9)$ \\
$\quad \begin{array}{l}\text { Non-infected group } \\
\text { 48 hours }\end{array}$ & $0.0(0.0-0.0)$ & $0.0(0.0-2.4)$ & $4.9(2.8-9.8)$ & $4(2-8)$ & $5(3-8)$ & $7(0-16)$ & $1.8(0.0-2.8)$ \\
Infected group & $0.0(0.0-2.9)$ & $3.9(0.0-6.3)$ & $3.7(1.8-6.4)$ & $24(3-61)$ & $14(8-21)$ & $14(0-49)$ & $2.1(0.0-3.5)$ \\
$\quad$ Non-infected group & $0.0(0.0-0.0)$ & $0.6(0.0-2.5)$ & $4.2(2.7-8.2)$ & $4(2-13)$ & $5(3-8)$ & $8(0-15)$ & $2.0(0.0-2.8)$ \\
\hline
\end{tabular}

Results are median (interquartile range)

IFN, interferon; IL, interleukin; TNF, tumour necrosis factor. 


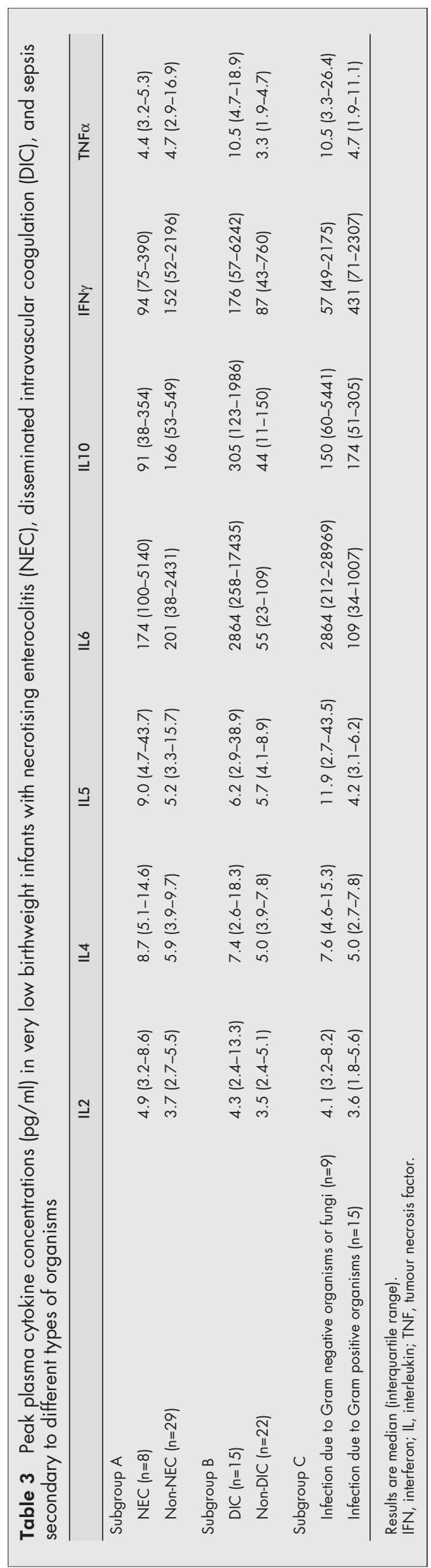

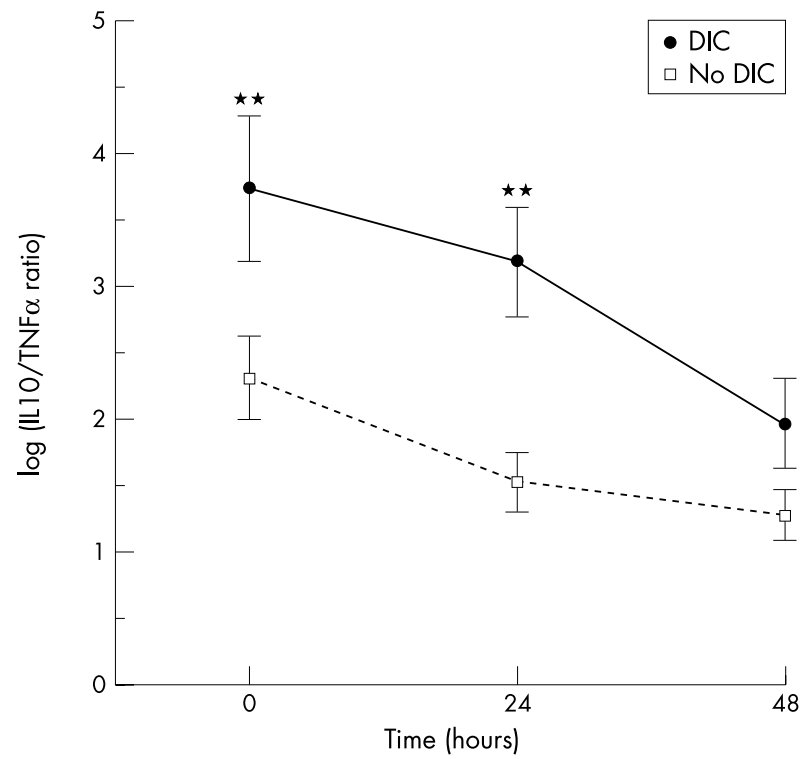

Figure 1 Comparison of interleukin (IL) 10/tumour necrosis factor (TNF) $\alpha$ ratios between septic infants with and without disseminated intravascular coagulation (DIC). The IL10/TNF $\alpha$ ratios at 0 and 24 hours were significantly higher in infected infants with DIC lexcluding the death case) than in those without. Double asterisks indicate a significant difference in the corresponding IL10/TNF $\alpha$ ratio between the two groups with $p<0.01$. Results are mean (SEM).

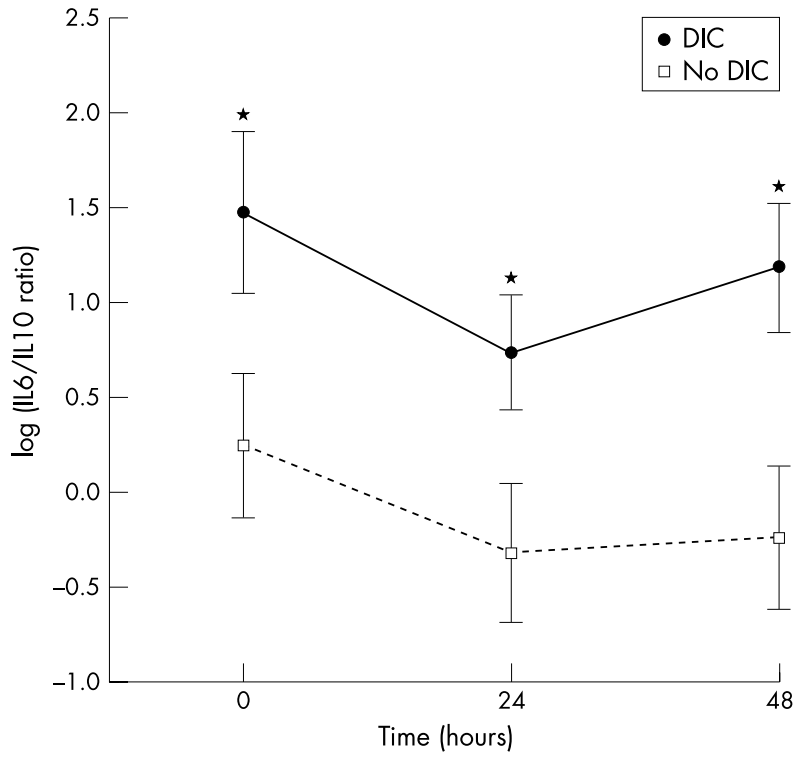

Figure 2 Comparison of interleukin(IL) 6/IL10 ratios between septic infants with and without disseminated intravascular coagulation (DIC). The IL6/IL 10 ratios at 0,24 , and 48 hours were significantly higher in infected infants with DIC (excluding the death case) than in those without. One asterisk indicates a significant difference in the corresponding IL6/IL10 ratio between the two groups with $p<0.05$. Results are mean (SEM).

IL4 or IL10. In humans, the release of IL10 by Th2 cells, B cells, and macrophages has been shown to be upregulated by circulating TNF $\alpha .^{20} \mathrm{IL} 10$ has the ability to suppress the synthesis of proinflammatory cytokines from $\mathrm{T}$ cells, leucocytes, and macrophages, ${ }^{21}{ }^{22}$ and effectively downregulates the proinflammatory response. In older children and adult patients with severe sepsis, high circulating levels of IL10 have been associated with septicaemic shock, exaggerated proinflammatory cytokine response, and the disease severity index. ${ }^{4324}$ Similarly, our data showed a significant correlation between 
IL6 and IL10 or TNF $\alpha$ in infected preterm infants and suggested that this counter-regulatory mechanism was probably operational very early in gestation.

The results of subgroup analysis indicate that patients with Gram negative or fungal septicaemia and those with DIC were more sick, and these patients elicited a more prominent proinflammatory as well as anti-inflammatory response than infants with Gram positive septicaemia and no DIC respectively. In contrast, the magnitude of cytokine response did not differ significantly in patients with NEC and in infected infants without NEC, suggesting that the severity of inflammatory response may be similar in these two groups of patients. A recent study reported that persistently high levels of IL10 or a high IL10/TNF $\alpha$ ratio were associated with poor prognosis and increased mortality in adult febrile patients. ${ }^{3}$ However, this association of high IL10 level with increased mortality was not confirmed in laboratory models of sepsis. In fact, exogenous administration of IL10 conferred protection against excessive proinflammatory cytokine production and mortality, ${ }^{9}{ }^{125}$ and neutralisation of endogenous IL10 with specific monoclonal antibodies resulted in increased proinflammatory cytokine levels and fatality rate. ${ }^{26}$ In accordance with the findings of van Dissel and co-workers, ${ }^{3}$ our data show a significant increase in IL10/TNF $\alpha$ ratio in more severely affected infants, but the ratio fell precipitously and began to normalise 48 hours after onset (fig 1). Thus, transiently elevated plasma IL10 levels or IL10/TNF $\alpha$ ratios did not necessarily indicate a poor outcome, as all except one of our patients with DIC survived. Although the IL10/TNF $\alpha$ ratios of the deceased patient declined after the start of treatment, this ratio remained relatively high at 48 hours ( $\log$ IL10/TNF $\alpha$ ratio 2.8). Further, Taniguchi $e t a l^{6}$ reported the correlation of high IL6/IL10 ratios and poor outcomes in adult patients with systemic inflammatory response syndrome. Our data also show a significantly higher IL6/IL10 ratio in patients with DIC. This ratio was disproportionally elevated in the deceased infant at presentation and continued to increase despite treatment. The change in the cytokine ratio in this case was mainly attributed to the continuous and substantial increase in IL6 $(5608 \mathrm{pg} / \mathrm{ml}$ at 48 hours), and also to a lesser extent the decrease in ILl0 ( $48.8 \mathrm{pg} / \mathrm{ml}$ at 48 hours). Thus our results point towards an exaggeration of proinflammatory activity coupled with an inadequate anti-inflammatory compensation in an infant whose clinical condition had been overwhelmed by severe sepsis. We speculate that both IL6 and IL10 play a crucial role in balancing the beneficial and adverse effects of the cytokine cascade during systemic infection.

In summary, our data suggest that the interaction between proinflammatory and anti-inflammatory cytokines plays an important role in the clinical manifestations and outcomes of systemic infection in preterm, VLBW patients. Our study also indicates that the counter-regulatory mechanisms between proinflammatory and anti-inflammatory pathways are probably operational early in gestation. High IL10/TNF $\alpha$ and IL6/IL10 ratios signify severe infection, but transiently elevated plasma IL10 concentrations or IL10/TNF $\alpha$ ratios at presentation do not necessarily imply poor prognosis, as most of our patients with DIC survived. We speculate that an exaggerated proinflammatory response in association with inadequate anti-inflammatory compensation may result in an adverse clinical outcome. Flow cytometric analysis, which requires only a small volume of blood ( $0.05 \mathrm{ml}$ plasma), is most suited for quantitative measurement of key cytokines in this population. The mechanism and role of cytokines in neonatal sepsis warrants further investigation, and immune modulation therapy such as monoclonal antibody to IL10 or individual cytokines should for the time being remain experimental.

\section{Authors' affiliations}

P C Ng, K Li, R P O Wong, K Chui, G Li, T F Fok, Department of Paediatrics, Prince of Wales Hospital, The Chinese University of Hong Kong, Hong Kong

E Wong, Centre of Clinical Trials and Epidemiological Research, Prince of Wales Hospital

\section{REFERENCES}

1 Romagnani S. T-cell subsets (Th1 versus Th2). Ann Allergy Asthma Immunol 2000;85:9-21

2 Zimmer S, Pollard V, Marshall GD, et al. Effects of endotoxin on the Th1/Th2 response in humans. Journal of Burns Care Rehabilitation 1996:17:491-6.

3 van Dissel JT, van Langevelde P, Westendorp RGJ, et al. Anti-inflammatory cytokine profile and mortality in febrile patients. Lance 1998;351:950-3.

4 Marchant A, Deviére J, Byl B, et al. Interleukin-10 production during septicaemia. Lancet 1994;343:707-8.

5 Kasai T, Inada K, Takakuwa T, et al. Anti-inflammatory cytokine levels in patients with septic shock. Res Commun Mol Pathol Pharmacol 1997;98:34-42

6 Taniguchi T, Koido Y, Aiboshi J, et al. Change in the ratio of interleukin-6 to interleukin-10 predicts a poor outcome in patients with systemic inflammatory response syndrome. Crit Care Med 1999;27:1262-4

7 Pinsky MR, Vincent JL, Deviére J, et al. Serum cytokine levels in human septic shock: relation to multiple-system organ failure and mortality. Chest 1993; 103:565-75.

8 Marty C, Misset B, Tamion F, et al. Circulating interleukin-8 concentrations in patients with multiple organ failure of septic and nonseptic origin. Crit Care Med 1994;22:673-9.

9 Gerard C, Bruyns C, Marchant A, et al. Interleukin 10 reduces the release of tumor necrosis factor and prevents lethality in experimental endotoxemia. J Exp Med 1993;177:547-50.

10 Howard M, Muchamuel T, Andrade S, et al. Interleukin 10 protects mice from lethal endotoxemia. J Exp Med 1993;177:1205-8.

11 Bone RC. Immunologic dissonance: a continuing evolution in our understanding of the systemic inflammatory response syndrome and the multiple organ dysfunction syndrome. Ann Intern Med 1996;125:690-1

12 Fisher CJ, Agosti JM, Opal SM, et al. Treatment of septic shock with the tumour necrosis factor: Fc fusion protein. N Engl J Med 1996;334: 1697-702

13 Mosmann TR. Cytokine secretion patterns and cross regulation of $T$ cell subsets. Immunol Res 1991;10:183-8.

14 Robinson DS, Hamid Q, Ying S, et al. Predominant Th2-like bronchoalveolar T-lymphocyte population in atopic asthma. N Engl J Med 1992;326:298-304

15 Rook GA, Hernandez-Pando R, Lightman SL. Hormones, peripherally activated prohormones and regulation of the Th1/Th2 balance. Immunol Today 1994; 15:301-3

16 Stern CM. Neonatal infection: neonatal immunology (Part 1). In: Roberton NRC, ed. Textbook of neonatology. 2nd ed. Edinburgh: Churchill Livingstone, 1992:925-41.

17 Ng PC, Cheng SH, Chui KM, et al. Diagnosis of late onset neonatal sepsis with cytokines, adhesion molecules, and $C$ reactive protein in preterm very low birthweight infants. Arch Dis Child Fetal Neonatal Ed 1997:77:F221-7.

18 Calanda T, Baumgartner JD, Grav GE, et al. The Swiss-Dutch J5 Immunoglobulin Study Group. Prognostic values of tumor necrosis factor/cachectin, interleukin-1, and interferon- $\alpha$, and interferon- $\gamma$ in the serum of patients with septic shock. J Infect Dis 1990;16:982-7.

19 Cannon JG, Tompkins RG, Gelfand JA, et al. Circulating interleukin-1 and tumor necrosis factor in septic shock and experimental endotoxin fever. J Infect Dis 1990;161:79-84

20 van der Poll T, Jansen J, Levi $M$, et al. Regulation of interleukin-10 release by tumor necrosis factor in humans and chimpanzees. J Exp Med 1994; 180:1985-8

21 Cassatella MA, Meda L, Bonora S, et al. Interleukin-10 (IL-10) inhibits the release of proinflammatory cytokines from polymorphonuclear leukocytes. Evidence for an autocrine role of tumor necrosis factor and IL-1 in mediating the production of IL-8 triggered by lipopolysaccharide. Exp Med 1993;178:2207-11.

22 Fiorentino DF, Zlotnik A, Mosmann TR, et al. Interleukin-10 (IL-10) inhibits cytokines by activated monocytes. J Immunol 1991;147:385422.

23 Marchant A, Alegre ML, Hakim A, et al. Clinical and biological significance of interleukin-10 plasma levels in patients with septic shock. J Clin Immunol 1995; 15:266-73.

24 Gomez-Jimenez J, Martin MC, Sauri R, et al. Interleukin-10 and the monocyte/macrophage-induced inflammatory response in septic shock. J Infect Dis 1995:171:472-75.

25 van der Poll T, Marchant A, Buurman WA, et al. Endogenous IL-10 protects mice from death during septic peritonitis. J Immunol 1995; 155:5397-401

26 Ishida H, Hastings R, Thompson-Snipes L, et al. Modified immunological status of anti-lL-10 treated mice. Cell Immunol 1993;148:371-84. 